OPEN ACCESS

Edited by: Paola Ulivi,

Istituto Scientifico Romagnolo per Io

Studio e la Cura dei Tumori

(IRCCS), Italy

Reviewed by:

Huaichao Luo,

Sichuan Cancer Hospital, China

Xingen Zhu

Second Affiliated Hospital of

Nanchang University, China

*Correspondence:

Liqun Wu

wula5810@126.com

Specialty section:

This article was submitted to Gastrointestinal Cancers,

a section of the journal

Frontiers in Oncology

Received: 19 October 2020 Accepted: 14 April 2021

Published: 10 June 2021

Citation:

Huo J, Wu L and Zang Y (2021)

Construction and Validation of a Reliable Six-Gene Prognostic Signature Based on the TP53 Alteration for Hepatocellular Carcinoma.

Front. Oncol. 11:618976. doi: 10.3389/fonc.2021.618976

\section{Construction and Validation of a Reliable Six-Gene Prognostic Signature Based on the TP53 Alteration for Hepatocellular Carcinoma}

Junyu Huo, Liqun $W^{*}{ }^{*}$ and Yunjin Zang

Liver Disease Center, The Affiliated Hospital of Qingdao University, Qingdao, China

Background: The high mutation rate of TP53 in hepatocellular carcinoma (HCC) makes it an attractive potential therapeutic target. However, the mechanism by which TP53 mutation affects the prognosis of HCC is not fully understood.

Material and Approach: This study downloaded a gene expression profile and clinicalrelated information from The Cancer Genome Atlas (TCGA) database and the international genome consortium (ICGC) database. We used Gene Set Enrichment Analysis (GSEA) to determine the difference in gene expression patterns between HCC samples with wildtype TP53 $(n=258)$ and mutant TP53 $(n=116)$ in the TCGA cohort. We screened prognosis-related genes by univariate Cox regression analysis and Kaplan-Meier (KM) survival analysis. We constructed a six-gene prognostic signature in the TCGA training group ( $n=184$ ) by Lasso and multivariate Cox regression analysis. To assess the predictive capability and applicability of the signature in HCC, we conducted internal validation, external validation, integrated analysis and subgroup analysis.

Results: A prognostic signature consisting of six genes (EIF2S1, SEC61A1, CDC42EP2, SRM, GRM8, and TBCD) showed good performance in predicting the prognosis of HCC. The area under the curve (AUC) values of the ROC curve of 1-, 2-, and 3-year survival of the model were all greater than 0.7 in each independent cohort (internal testing cohort, $n=181$; TCGA cohort, $n=365$; ICGC cohort, $n=229$; whole cohort, $n=594$; subgroup, $n=9$ ). Importantly, by gene set variation analysis (GSVA) and the single sample gene set enrichment analysis (SSGSEA) method, we found three possible causes that may lead to poor prognosis of HCC: high proliferative activity, low metabolic activity and immunosuppression. 
Conclusion: Our study provides a reliable method for the prognostic risk assessment of HCC and has great potential for clinical transformation.

Keywords: hepatocellular carcinoma, TP53, prognostic, signature, biomarker

\section{BACKGROUND}

Hepatocellular carcinoma (HCC) is a major cause of cancer mortality due to its high incidence rate, high recurrence, and limited molecular targeted therapeutic options $(1,2)$. Thus, there is an urgent need to discover novel biomarkers and design novel therapeutic strategies for HCC.

TP53 mutations occur in almost every type of cancer at rates from 38\%-50\%. Additionally, it has been shown that TP53 mutations were more frequent in cancer patients with lower survival rates among all cancer types studied (3). Wild-type TP53 monitors abnormal activities in cells, senses cell pressure or damage, and prevents the proliferation of damaged cells (4). However, under TP53 mutation, cells exhibiting DNA damage are capable of escaping apoptosis and transforming into carcinoma cells. In addition, TP53 mutant proteins lose their wild-type function and accumulate in the nucleus, a significant hallmark of malignant tumors (5). A large sample study of 10225 cancer patients found that TP53 mutations were more frequent in cancer patients with lower survival rates among all 32 cancer types studied (6). Professor Donhower's (6) study also found that in most TP53 mutant tumors, oncogene amplification increased, and tumor suppressor genes were deeply deleted. The expression patterns of RNA, miRNA and protein in TP53-mutated tumors are different from those in nonmutated tumors. The expression of cell cycle progression genes and proteins in TP53-mutated tumors is enhanced.

The TP53 mutation process is the most common mutation process in HCC. This gene is critical for maintaining the stable properties of the genome. Its loss of function can lead to a centrosome amplification process, aneuploid cell proliferation process and chromosome instability $(7,8)$. Some related reports showed that the TP53 mutation process was not only related to the prognosis of HCC (9) but also correlated with serum alphafetoprotein (AFP), clinical stage, vascular invasion, tumor differentiation and Child-Pugh grade (10-13). Therefore, TP53 mutation has a profound impact on the genomic structure, expression and clinical prospects of HCC. Understanding the effect of TP53 on the pathogenesis of HCC is critical to develop more effective treatments for HCC.

The more we learn about TP53, the more we can understand the basic biology of HCC and develop better treatments. In this study, we speculated that the change in the RNA expression pattern caused by TP53 mutation may be an important reason

Abbreviations: HCC, hepatocellular carcinoma; TCGA, The Cancer Genome Atlas; ICGC, International Cancer Genome Consortium; GSEA, Gene Set Enrichment Analysis; KM, Kaplan-Meier; DEGs, differential expressed genes; GSVA, Gene Set Variation Analysis; ssGSEA, single sample gene set enrichment analysis; KEGG, Kyoto Encyclopedia of Genes and Genomes; ROC, receiver operating characteristic; AUC, area under the curve; OS, overall survival; LASSO, Least absolute shrinkage and selection operator; FDR, false discovery rate. for the difference in prognosis. Based on this hypothesis, we have successfully developed an approach capable of predicting accurate HCC prognosis. Considering the accuracy and universal applicability of the model, the six genes included may be likely targets to treat HCC.

\section{MATERIAL AND APPROACH}

\section{Research Object}

The clinical and transcriptome (fragments per kilobase of transcript per million (FPKM)) data of 374 HCC samples were acquired from The Cancer Genome Atlas (TCGA-LIHC) website (https://portal.gdc.cancer.gov/projects/TCGA-LIHC). Among them, there were 116 cases of TP53 mutation and 258 cases of wild-type TP53, and a detailed list of TP53-mutated samples was obtained from the cBioPortal website (https://www.cbioportal. org/). A total of 365 cases had complete prognosis information. We acquired the gene expression profile and clinic-related data of the LIRI-JP dataset from the International Genome Consortium (ICGC) database (https://dcc.icgc.org/releases/ current/Projects/LIRI-JP) for external validation $(n=229)$. TCGA and ICGC were all based on the Illumina HiSeq platform. The work here fully complied with the publication guidelines of TCGA and ICGC. Our research was based on public databases; therefore, further approval from the local ethics committee was not needed. The detailed clinical information of the TCGA and ICGC cohorts is shown in Table $\mathbf{1}$.

\section{Gene Set Enrichment Analysis}

To determine the difference in gene expression patterns between HCC samples with wild-type TP53 $(n=258)$ and mutant TP53

TABLE 1 | The clinical information of TCGA and ICGC cohort.

\begin{tabular}{lcc}
\hline & TCGA & ICGC \\
\hline $\begin{array}{l}\text { Survival status } \\
\text { Alive }\end{array}$ & \\
$\quad$ Dead \\
$\begin{array}{l}\text { Gender } \\
\quad \text { Female }\end{array}$ & 235 & 187 \\
$\quad$ Male & 130 & 42 \\
Age & & 61 \\
$\quad<=65$ & 108 & 168 \\
$\quad>65$ & 231 & 88 \\
Stage-TNM & & 141 \\
I-II & 219 & 139 \\
III-IV & 120 & 90 \\
Histologic grade & & \\
$\quad$ G1-2 \\
G3-4
\end{tabular}


$(n=116)$ in the TCGA-LIHC cohort, we performed gene set enrichment analysis. The gene set file (h.all.v7.1.symbols.gmt) was used as the reference gene group. The threshold value was set as $\mathrm{P}<0.05$ and FDR $<0.25$.

\section{Screening of Prognosis-Related Genes}

We screened prognosis-related genes by univariate Cox regression analysis and Kaplan-Meier (KM) survival analysis. The genes with $\mathrm{P}$ values less than 0.05 obtained by the two methods were used for subsequent study.

\section{Construction and Validation of Prognostic Model}

To improve the generalization ability of the established prognostic model, 365 HCC cases with complete prognostic information were randomly divided into two independent cohorts: a training cohort $(\mathrm{n}=184)$ and a validation cohort $(\mathrm{n}=181)$. The aforementioned process was implemented using the R package 'Caret'. In the training cohort $(\mathrm{n}=184)$, we used least absolute shrinkage and selection operator (LASSO) penalty Cox regression analysis to further select prognostic genes. The LASSO algorithm with penalty parameter tuning performed via 10 -fold cross-validation was applied to exclude genes that may be highly correlated with other genes. We performed 100010 -fold cross-validations of data sets and selected genes with more than 900 repetitions $(14,15)$. A subset of genes was determined by shrinking the regression coefficient using a penalty proportional to their size. The genes with nonzero regression coefficients were retained for subsequent multivariate Cox regression analyses (16, 17). Finally, the regression coefficient obtained by multivariate Cox regression analysis was multiplied by the expression level of each gene to construct a prognostic model. All subjects were divided into two risk groups according to the median risk of the training cohort, and individuals with a risk score higher or lower than the median risk were divided into high-risk and low-risk groups, respectively. The Kaplan-Meier (KM) survival curve and the receiver operating characteristic (ROC) curve were used to assess the predictive ability of the model. To evaluate whether the prognostic model is independent of the traditional clinical features, we conducted univariate and multivariate Cox regression analyses of the prognostic model and the traditional clinical features. For internal and external validation, the testing cohort $(n=181)$, TCGA cohort $(n=365)$, external validation cohort (ICGC, $\mathrm{n}=229$ ), whole cohort (all included samples, $\mathrm{n}=$ 594) and subgroup $(n=9)$ survival analysis were adopted to validate the predictive capability and applicability exhibited by the prognostication signal in HCC.

LASSO regression was implemented using the 'glmnet' $\mathrm{R}$ package. The survival curve was generated using Kaplan-Meier survival analysis and visualized using the R package 'survminer', and data were analyzed using the log-rank test. The ROC curves were drawn, and the corresponding AUC values were calculated using the R package 'timeROC'.

\section{Gene Set Variation Analysis}

We first used the 'Limma' R package to identify genes with differential expression (DEGs) between the high-risk group and the low-risk group in the TCGA and ICGC cohorts. Adjusted P value $<0.05$ and absolute value of fold change $(\mathrm{FC})>1$ were suggested to indicate statistical significance. Then, we performed gene set variation analysis (GSVA) on the DEGs to identify prognosis-associated signaling pathways associated with the signature using the 'GSVA' $\mathrm{R}$ package. For GSVA, we chose Kyoto Encyclopedia of Genes and Genomes (KEGG) pathways as the reference and adj $P$ value $<0.05$ as the cutoff to screen significantly altered pathways.

\section{Correlation Analysis Between Signature and Immunity}

We conducted single sample gene set enrichment analysis (ssGSEA, 29 immune-related gene sets representing immune cell type, function, and pathway) to quantify the activity or enrichment levels of immune cells, functions, or pathways in the high- and lowrisk samples from TCGA and ICGC, respectively. The normalized enrichment score (NES) calculated from ssGSEA was calculated using the 'GSVA' R package. We used independent-samples t tests to explore the differences in immune infiltration levels and immune function between the high- and low-risk groups, and $\mathrm{P}<0.05$ was suggested to indicate statistical significance.

\section{RESULTS}

\section{Gene Set Enrichment Analysis of TP53 in the TCGA Database}

A brief workflow for this study is shown in Figure 1. Compared to the unaltered group, the patients with TP53 mutation had both significantly poorer overall survival and disease-free survival (Figures 2A, B). We carried out GSEA of HCC samples from wild-type TP53 $(\mathrm{n}=258)$ and mutant TP53 ( $\mathrm{n}=$ 116) samples. The results showed that 7 of the hallmark gene sets $(n=50)$ were significantly enriched in TP53-mutated HCC samples (Table 2, Figure 2C). We extracted 1048 genes from these 7 gene sets for subsequent analysis.

\section{Identification of Prognosis-Related Genes}

By univariate Cox regression analysis and Kaplan-Meier (KM) survival analysis (patients were classified into low and high expression groups based on median gene expression data), a total of 51 genes were screened out, of which 49 genes were unfavorable to prognosis $(\mathrm{HR}>1)$ and 2 genes were favorable to prognosis $(\mathrm{HR}<1)$ (Table 3$)$.

\section{Construction of a Six-Gene Prognostication Signal in the Training Cohort}

A total of 184 HCC patients were enrolled in the training set. The flowchart of the prognosis-scoring model construction process is shown in Figure 3A. The aforementioned 51 genes were further reduced by Lasso penalty Cox regression analysis, and we subsampled the dataset 1000 times with 10-fold cross-validation. After that, 41 genes with zero Lasso regression coefficients were excluded with the optimal value of $\log \lambda(-3.2)$, and 10 genes with 


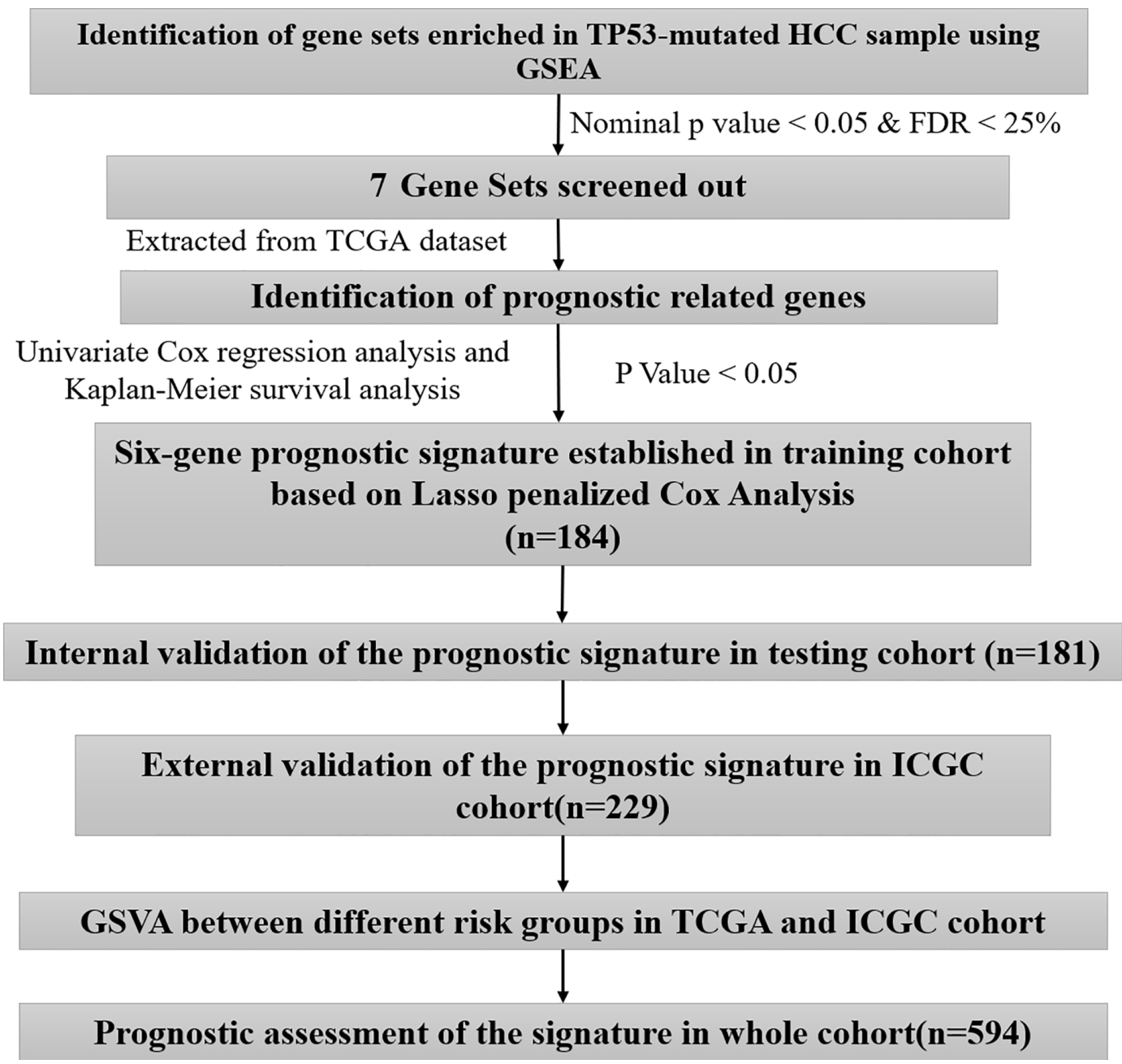

FIGURE 1 | The workflow chart of this study.

nonzero Lasso regression coefficients were included in the multivariate Cox regression analysis. A novel risk score was calculated by multiplying the gene expression of each gene and its corresponding coefficient, which was obtained by multivariate Cox regression analysis. Risk score $=\mathrm{EIF} 2 \mathrm{~S}^{\star} 0.1055+\mathrm{SEC} 1 \mathrm{~A} 1^{\star}$ $0.0082+\mathrm{CDC}_{2} \mathrm{EP} 22^{\star} 0.2127+\mathrm{SRM}^{\star} 0.0155+\mathrm{GRM}^{\star} 0.1344+$ $\mathrm{TBCD}^{\star} 0.0600$. The patients were grouped into high-risk or lowrisk categories using the median risk score of the training series as the cutoff point (0.81). There was a significant difference in RNA expression level between tumor $(\mathrm{n}=374)$ and normal tissues $(\mathrm{n}=$ 50) of the 6 genes in the signature (Figure 3B). Higher levels of the six mRNAs were associated with decreased overall survival (OS) in the TCGA cohort $(n=365)$ (Figure 3C). The OS of HCC patients in the cohort exhibiting high risk was significantly lower than that of the cohort exhibiting low risk (Figure 4A, P < 0.001). ROC curve analysis demonstrated the predictive ability of the risk score for 1-, 2and 3-year OS, with areas under the curve (AUCs) of 0.740, 0.757 and 0.756, respectively (Figure 4B). Univariate and multivariate Cox regression analyses showed that only the risk score of the 6-gene signature was an independent prognostic element (Figure 4C).

\section{Internal Validation of the Prognostic Signature in the Testing and Entire TCGA Cohort}

An identical risk score formula and cutoff value determined from the training set were used to analyze the testing cohort. Consistent with the results in the training set, the KM curves of the testing sets showed that the cohort exhibiting high risk had a worse prognosis than the low-risk group (Figure 4D). The AUC values for the signature predicting OS at 1,2 and 3 years were $0.770,0.736$ and 0.710 , respectively (Figure 4E), indicating good prediction accuracy. From the results of both univariate and multivariate Cox regression analyses, the risk score was found to be an independent poor prognostic indicator of HCC (Figure 4F). To validate the accuracy of the risk model, we analyzed the model in the entire TCGA cohort, and the results were in line with the above-described results (Figures 4G-I). 


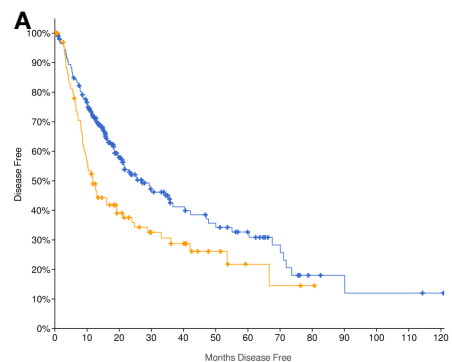

Disease Free
- Unalated group
TP53

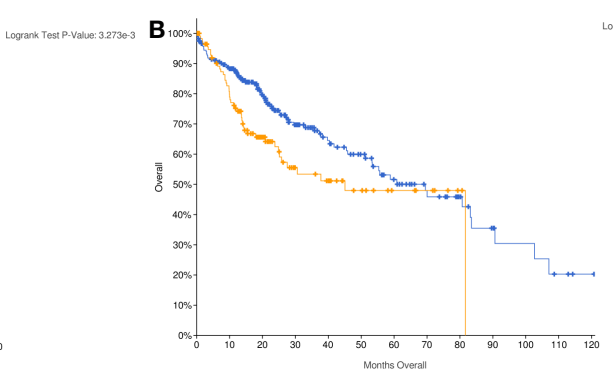

Overall
- Unatered group
TP53
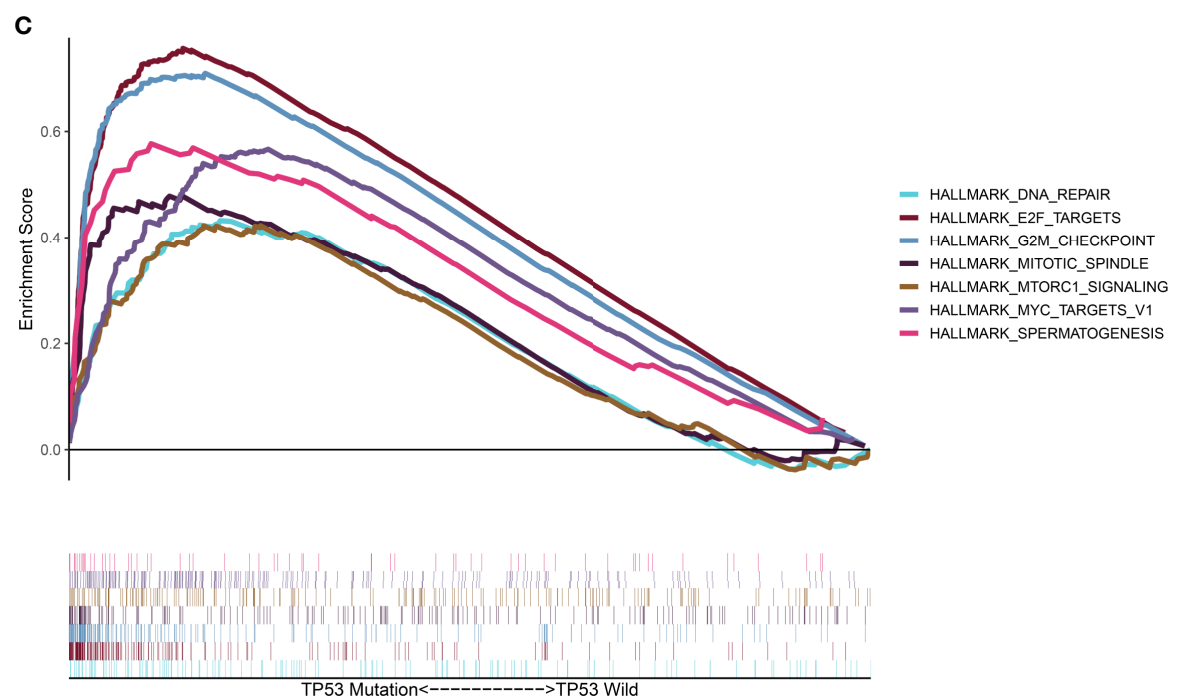

FIGURE 2 | Gene Set Enrichment Analysis(GSEA) in TP53-mutated HCC sample (A) The Kaplan-Meier survival curve regarding the TP53 and disease free survival (B) The Kaplan-Meier survival curve regarding the TP53 and overall survival (C) The 7 gene sets identified by GSEA in TP53-mutated HCC sample.

\section{External Validation of the Prognostic Signature in the ICGC Cohort}

For the in-depth assessment of the reliability of the prognostication model, an external dataset from the ICGC database was employed to verify the six-gene signature. The risk score was determined for each case according to the six-gene signature, and the 229 patients were divided into a high- or low-risk cohort based on their risk score. The Kaplan-Meier plot indicated that patients in the cohort exhibiting high risk had significantly shorter overall survival than those in the low-risk group (Figure 5A). The AUCs at 1, 2 and 3 years were 0.835 , 0.786 , and 0.809 , respectively, indicating that the risk score played a significant role in the prediction of prognosis (Figure 5B). As shown in Figure 5C, the six-gene expression level was increased significantly in the high-risk group. The risk of mortality tended to rise along with the risk score (Figures 5D, E). Both univariate and multivariate analyses suggested that the risk score could be used as an independent prognostic indicator (Figures 5F, G).

\section{Gene Set Variation Analysis Between Different Risk Groups}

We identified the differentially expressed genes (DEGs) between the high-risk and low-risk groups in the ICGC and TCGA

TABLE 2 | The 7 gene sets identified by GSEA in TP53-mutated HCC sample.

\begin{tabular}{|c|c|c|c|c|c|}
\hline NAME & SIZE & ES & NES & NOM p-val & FDR q-val \\
\hline HALLMARK_E2F_TARGETS & 184 & 0.75597 & 2.193104 & 0 & 0.001166 \\
\hline HALLMARK_G2M_CHECKPOINT & 181 & 0.709121 & 2.109249 & 0.002 & 0.001891 \\
\hline HALLMARK_SPERMATOGENESIS & 62 & 0.577112 & 2.012077 & 0.002101 & 0.007034 \\
\hline HALLMARK_MYC_TARGETS_V1 & 198 & 0.566704 & 1.822196 & 0.035052 & 0.037724 \\
\hline HALLMARK_MITOTIC_SPINDLE & 183 & 0.478536 & 1.662072 & 0.038911 & 0.096263 \\
\hline HALLMARK_MTORC1_SIGNALING & 191 & 0.423001 & 1.655123 & 0.041485 & 0.083437 \\
\hline HALLMARK_DNA_REPAIR & 143 & 0.432414 & 1.648957 & 0.035565 & 0.074602 \\
\hline
\end{tabular}


TABLE 3 | The prognostic related gene list.

\begin{tabular}{|c|c|c|c|c|c|}
\hline Gene & KM & HR & HR.95L & HR.95H & coxPvalue \\
\hline SLC38A1 & 0.003165 & 1.037392 & 1.014199 & 1.061115 & 0.001462 \\
\hline TXNL4A & 0.001014 & 1.017909 & 1.006116 & 1.02984 & 0.002832 \\
\hline PGK1 & 0.000866 & 1.00361 & 1.00133 & 1.005895 & 0.001899 \\
\hline SORBS2 & 6.72E-05 & 0.969257 & 0.945723 & 0.993376 & 0.012776 \\
\hline $\mathrm{PHB}$ & 0.002315 & 1.010017 & 1.003383 & 1.016693 & 0.00303 \\
\hline STIP1 & 0.00013 & 1.023625 & 1.015808 & 1.031503 & 2.37E-09 \\
\hline SNRPB2 & 0.000277 & 1.042278 & 1.021676 & 1.063296 & 4.80E-05 \\
\hline AMD1 & 1.14E-05 & 1.071158 & 1.042441 & 1.100666 & 7.13E-07 \\
\hline GAPDH & 1.52E-05 & 1.00039 & 1.000175 & 1.000604 & 0.000365 \\
\hline CKS1B & 0.00224 & 1.011813 & 1.004784 & 1.018892 & 0.000961 \\
\hline CCT7 & 0.000401 & 1.014353 & 1.008173 & 1.020571 & 4.86E-06 \\
\hline PSMA3 & 0.003753 & 1.01925 & 1.008159 & 1.030463 & 0.000636 \\
\hline CDC25B & 0.002845 & 1.023348 & 1.011816 & 1.03501 & 6.56E-05 \\
\hline TPI1 & 0.000173 & 1.001565 & 1.000473 & 1.002658 & 0.004961 \\
\hline EIF2S1 & 0.003714 & 1.132416 & 1.08095 & 1.186332 & 1.61E-07 \\
\hline VDAC1 & 0.005192 & 1.007353 & 1.002668 & 1.012061 & 0.002071 \\
\hline ASNS & 0.003391 & 1.02885 & 1.006893 & 1.051285 & 0.009763 \\
\hline EIF2S2 & 0.000218 & 1.023907 & 1.011755 & 1.036205 & 0.000105 \\
\hline SEC61A1 & 4.58E-06 & 1.008706 & 1.004549 & 1.012879 & 3.88E-05 \\
\hline CDK4 & 5.22E-05 & 1.018012 & 1.010166 & 1.02592 & 6.13E-06 \\
\hline AK2 & 0.005891 & 1.038733 & 1.01649 & 1.061463 & 0.00058 \\
\hline YKT6 & 0.000413 & 1.018644 & 1.00684 & 1.030588 & 0.001896 \\
\hline CDC42EP2 & 0.000296 & 1.091183 & 1.034936 & 1.150487 & 0.001231 \\
\hline NME1 & 0.001137 & 1.004218 & 1.00077 & 1.007676 & 0.016442 \\
\hline PSMD13 & 0.000882 & 1.017424 & 1.006653 & 1.02831 & 0.001467 \\
\hline CDCA8 & 2.39E-06 & 1.123353 & 1.085664 & 1.16235 & $2.38 \mathrm{E}-11$ \\
\hline CDC20 & 3.85E-06 & 1.027371 & 1.01811 & 1.036715 & 5.07E-09 \\
\hline SNRPD1 & 0.000174 & 1.026441 & 1.010183 & 1.042961 & 0.001357 \\
\hline RAN & 2.09E-05 & 1.010095 & 1.006302 & 1.013904 & $1.68 \mathrm{E}-07$ \\
\hline SNRPA & 8.27E-05 & 1.014106 & 1.005375 & 1.022913 & 0.001499 \\
\hline E2F2 & 5.30E-05 & 1.684961 & 1.322284 & 2.147112 & 2.45E-05 \\
\hline EFNA5 & 0.001057 & 1.082642 & 1.013996 & 1.155935 & 0.01751 \\
\hline $\mathrm{ERH}$ & 0.000486 & 1.012517 & 1.005591 & 1.019491 & 0.000383 \\
\hline PPIA & 0.000415 & 1.00327 & 1.001701 & 1.00484 & 4.32E-05 \\
\hline LDHA & 8.70E-05 & 1.003787 & 1.002332 & 1.005243 & $3.25 \mathrm{E}-07$ \\
\hline SRM & 0.001693 & 1.010772 & 1.004765 & 1.016814 & 0.000427 \\
\hline DTYMK & 1.32E-05 & 1.032033 & 1.017913 & 1.04635 & 7.26E-06 \\
\hline POLE4 & 0.018558 & 1.016728 & 1.004422 & 1.029185 & 0.007581 \\
\hline NT5C & 0.001771 & 1.009348 & 1.000365 & 1.018411 & 0.041354 \\
\hline RBX1 & 0.006273 & 1.021113 & 1.005411 & 1.037061 & 0.008231 \\
\hline GRM8 & 0.000359 & 1.228372 & 1.108225 & 1.361545 & 8.98E-05 \\
\hline ADAD1 & 0.001756 & 1.77152 & 1.102922 & 2.845424 & 0.018023 \\
\hline RANBP1 & 7.04E-05 & 1.01552 & 1.008045 & 1.023051 & 4.40E-05 \\
\hline SORD & 0.002689 & 0.993698 & 0.989909 & 0.997501 & 0.001178 \\
\hline PTGES3 & 2.89E-05 & 1.009964 & 1.004996 & 1.014956 & 8.11E-05 \\
\hline RAE1 & 0.000744 & 1.115734 & 1.066832 & 1.166877 & 1.68E-06 \\
\hline TBCD & 0.000116 & 1.056685 & 1.018552 & 1.096244 & 0.00328 \\
\hline PSMA7 & 0.001897 & 1.004442 & 1.001317 & 1.007576 & 0.005301 \\
\hline EEF1E1 & 6.14E-07 & 1.045183 & 1.016904 & 1.074247 & 0.00159 \\
\hline CTSC & 0.000925 & 1.005762 & 1.001276 & 1.010267 & 0.011757 \\
\hline POLR2G & 0.001017 & 1.023782 & 1.011387 & 1.03633 & 0.000156 \\
\hline
\end{tabular}

datasets (Figures 6A, C). Subsequently, we made use of these DEGs to perform gene set variation analysis (GSVA). KEGG pathway activities were scored per sample by GSVA between the different risk groups. We found a significant decrease in metabolism-related signaling pathway GSVA scores in the cohort exhibiting high risk (Tables 4, 5 and Figures 6B, D). Furthermore, oocyte meiosis-associated genes exhibited higher enrichment scores in the cohort demonstrating high risk (Tables 4, 5 and Figures 6B, D).

\section{Differences in the Immune Landscape Between Different Risk Groups}

We attempted to explore cases involving differences in prognosis in different risk groups from the perspective of the immune microenvironment. We used the ssGSEA score to quantify the activity or enrichment levels of immune cells and functions in the HCC samples (Figures 7A, D). Similar results were obtained from two independent cohorts. In terms of immune cell infiltration, the infiltration abundance of aDCs, iDCs, 


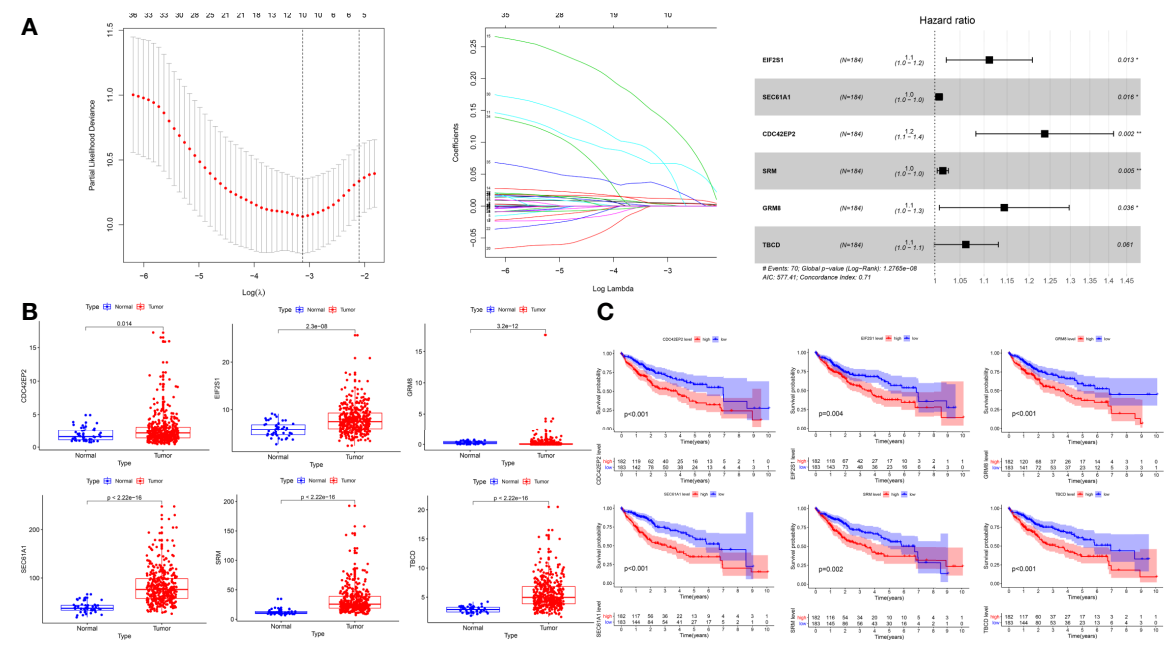

FIGURE 3 | Construction of the prognostic model in the training cohort (A) The building process of the six-gene prognostic signature (B) The expression level of the six genes between tumor and normal tissues (C) The Kaplan-Meier survival curve of the expression level of the six genes and overall survival.
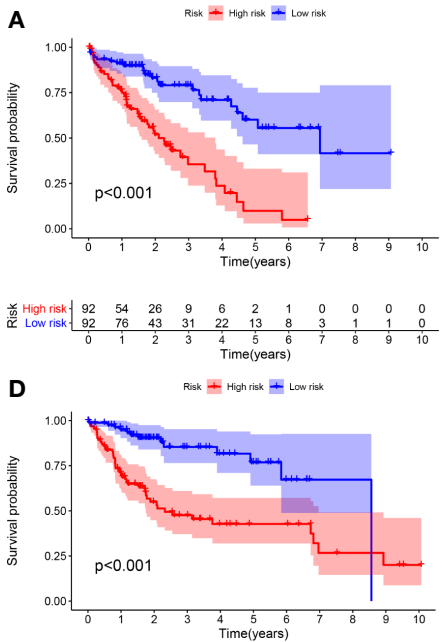

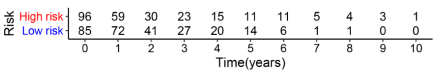

G

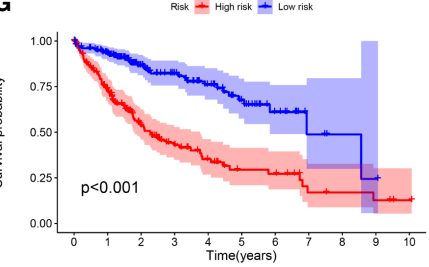

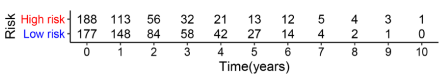

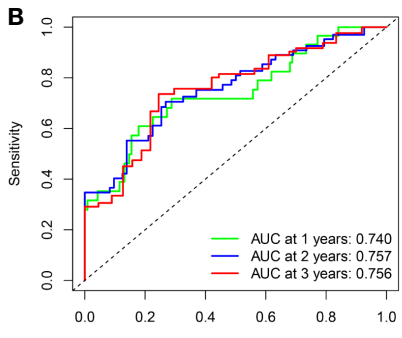

1-Specificity
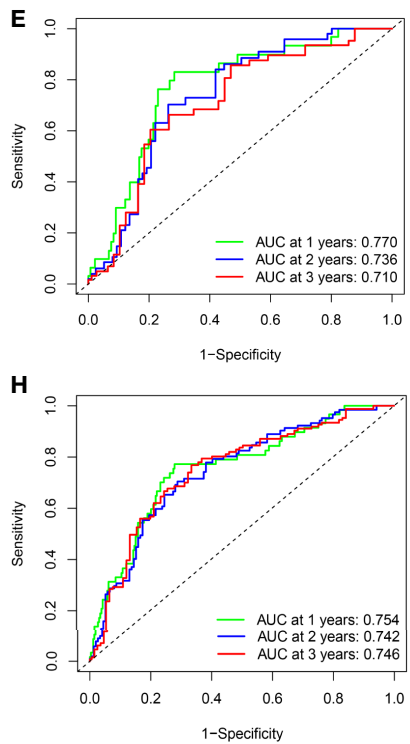

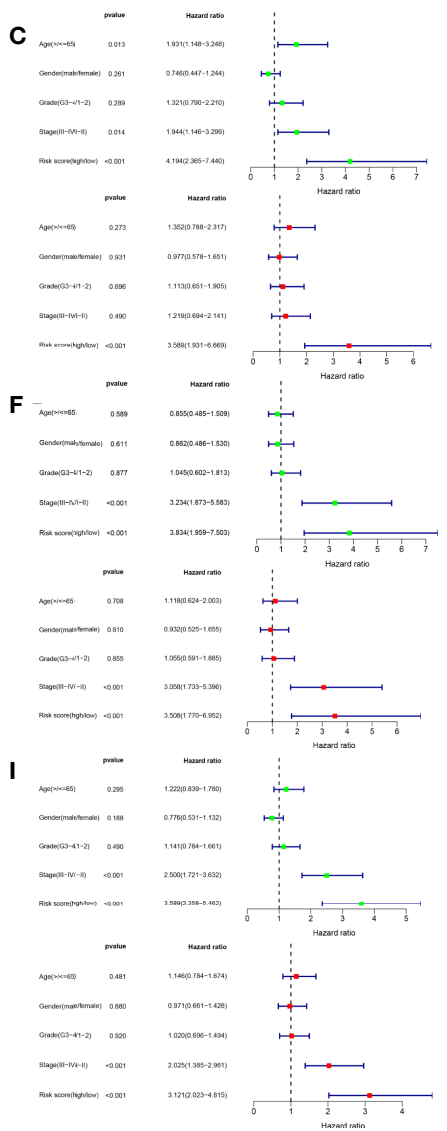

FIGURE 4 | Prognostic assessment of the prognostic model in the TCGA cohort (A-C) The Kaplan-Meier survival analysis, time-dependent ROC analysis, and independence analysis of the signature for predicting the overall survival of patients in the training cohort ( $\mathrm{n}=184)$ (D-F) The Kaplan-Meier survival analysis, time-dependent ROC analysis, and independence analysis of the signature for predicting the overall survival of patients in the testing cohort( $\mathrm{n=181)}$ (G-I) The Kaplan-Meier survival analysis, time-dependent ROC analysis, and independence analysis of the signature for predicting the overall survival of patients in the entire TCGA cohort(n=365). Green represents univariate analysis, and red represents multivariate analysis. 
A
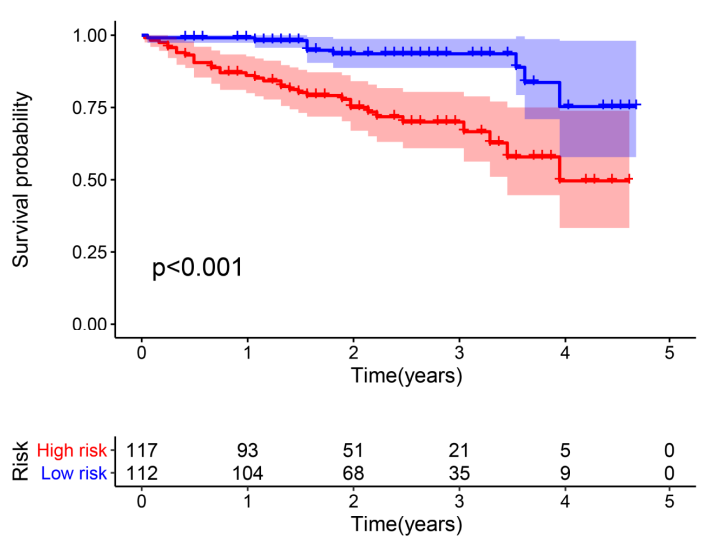

B

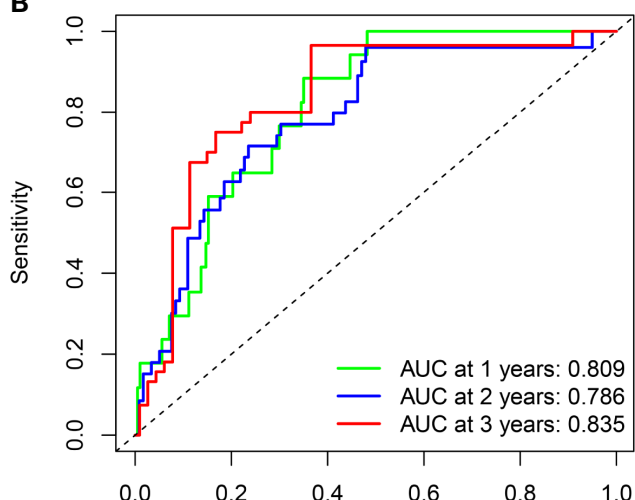

F

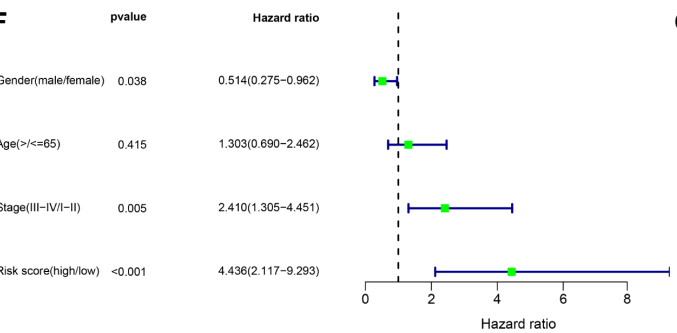

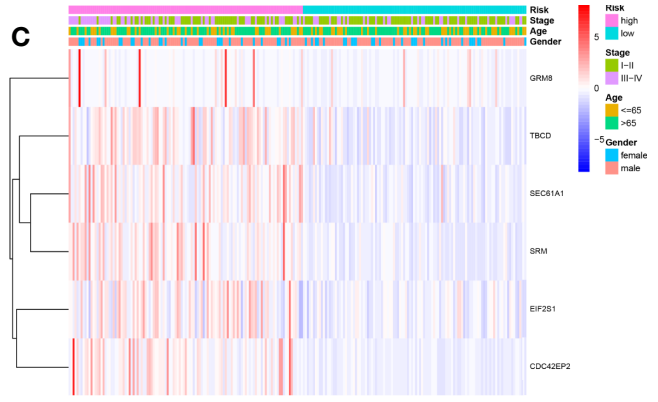

D

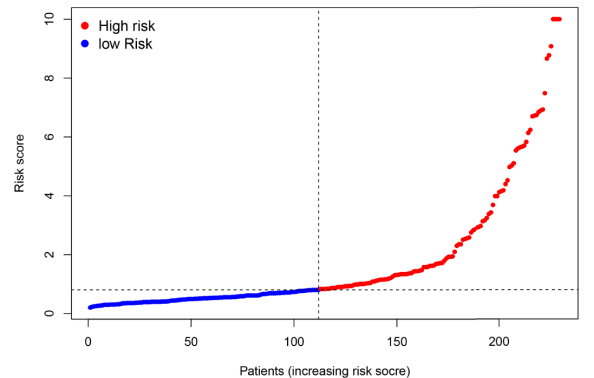

E

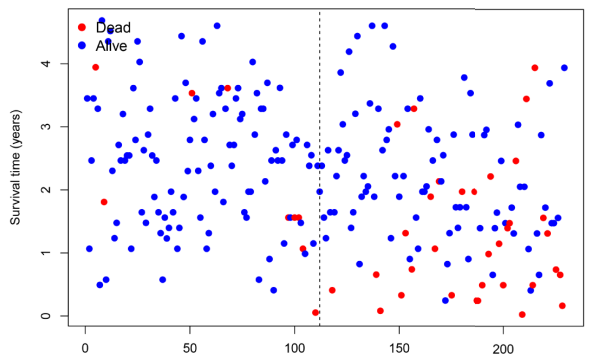

G
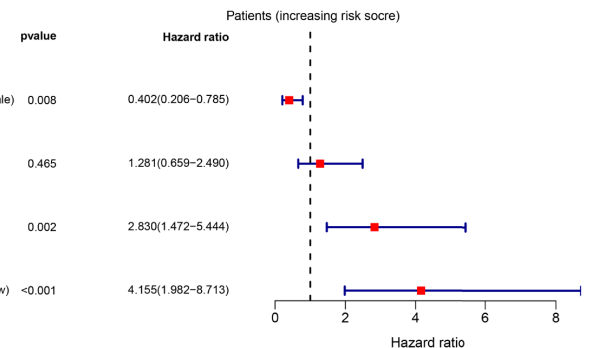

FIGURE 5 | Prognostic assessment of the prognostic model in the ICGC cohort (A, B) The Kaplan-Meier survival analysis and time-dependent ROC analysis of the signature for predicting the overall survival of patients in the ICGC cohort $(n=229)$ (C) The heatmap of the six-gene signature (D) The survival status of patients (E) The distribution of the risk score (F, G) Univariate and multivariate regression analyses of the relation between the risk score and clinicopathological characteristics regarding the overall survival in the ICGC cohort (green represents univariate analysis, and red represents multivariate analysis).

macrophages, NK cells, and Tregs in the cohort exhibiting high risk was significantly higher than that in the low-risk group (Figures 7B, E). In terms of immune function, the results revealed that the expression of immune checkpoints and $\mathrm{MHC}$ class I-related gene sets was upregulated in the high-risk group, while the expression of type I and II IFN response-related gene sets was upregulated in the cohort exhibiting low risk (Figures 7C, F). We also detected the expression levels of PD1 (PDCD1), PDL1, TIGIT, CTLA4 and LAG3 in the high-risk and low-risk groups. This study reported that the risk score positively correlated with the expression levels of PD1 (PDCD1), PDL1,
TIGIT, CTLA4 and LAG3. The expression levels of these five common immune checkpoints were all upregulated in the highrisk cohort (Figures 8A-C).

\section{Integrated Analysis of the Prognostication Signal in the Whole Group}

A total of 594 cases were covered in this pooled analysis. The results were consistent with the conclusion from a previous study. In terms of OS, patients exhibiting high risk had a poorer OS than patients exhibiting low risk (Figure 9A). The AUC values of the 1-year, 2-year and 3-year overall 
A

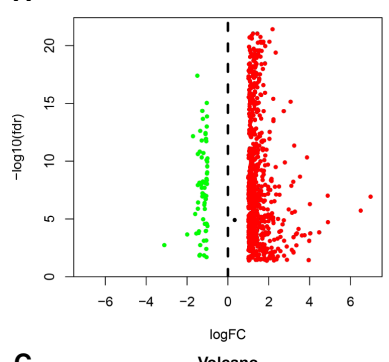

C

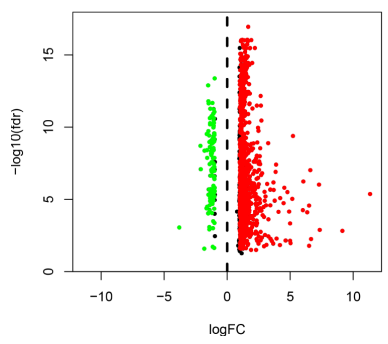

B

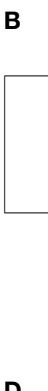

D
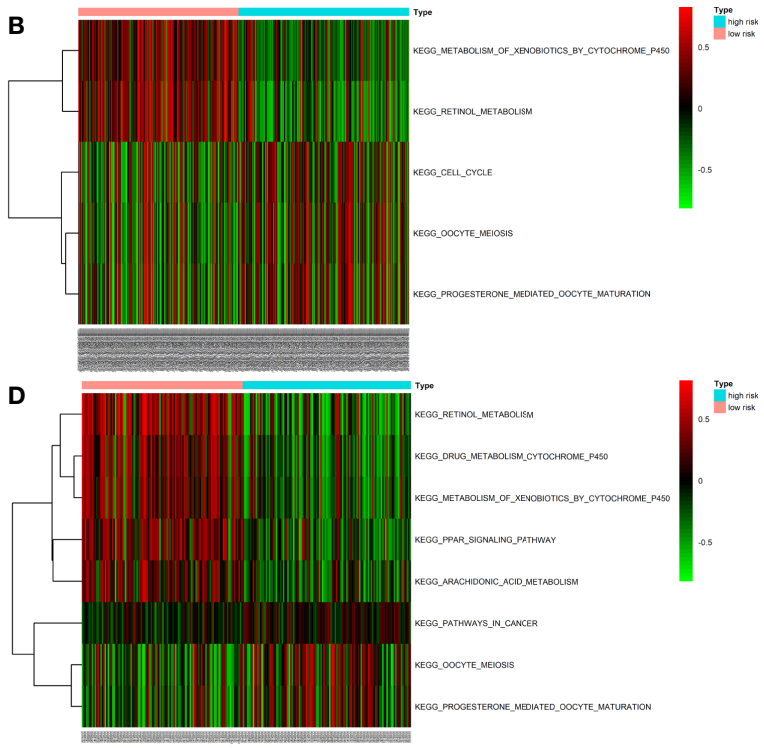

FIGURE 6 | Gene Set Variation Analysis between high- and low risk groups in the TCGA and ICGC cohort (A) Differentially expressed genes (DEGs) between highand low-risk groups in the TCGA cohort (B) Gene Set Variation Analysis between high- and low risk groups in the TCGA cohort (C) Differentially expressed genes (DEGs) between high- and low-risk groups in the ICGC cohort (D) Gene Set Variation Analysis between high- and low risk groups in the ICGC cohort.

survival rates of patients predicted by the risk score were 0.761 , 0.750, and 0.772, respectively (Figure 9B). Univariate and multivariate Cox regression analyses revealed that a high risk score indicated poor clinical outcome in HCC patients (Figure 9C). In addition, TP53 and TNM stage were significant prognostic elements (Figures 9D, F), and a positive correlation was found between risk score and TNM stage (Figure 9E). Patients with TP53 mutations had higher risk scores (Figure 9G).

\section{Subgroup Survival Analysis}

To validate the general applicability of the prognostication signal in HCC, we grouped the 594 patients according to their clinical characteristics. In each subgroup, cases exhibiting high risk were compared with those exhibiting low risk. The prognostication of cases with high risk was noticeably worse than that of cases with low risk in each subgroup (Figures 10A-D). These results confirmed that the risk score had good stratification ability for the prognosis of HCC.

TABLE 4 | Gene Set Variation Analysis between high- and low risk groups in the TCGA cohort.

\begin{tabular}{|c|c|c|c|c|c|c|}
\hline NAME & $\log F C$ & AveExpr & $\mathbf{t}$ & P.Value & adj.P.Val & B \\
\hline KEGG_METABOLISM_OF_XENOBIOTICS_BY_CYTOCHROME_P450 & 0.252025 & 0.049534 & 9.814887 & 2.15E-20 & $3.44 \mathrm{E}-19$ & 35.49329 \\
\hline KEGG_RETINOL_METABOLISM & 0.287397 & 0.033523 & 9.701779 & $5.24 \mathrm{E}-20$ & $7.85 \mathrm{E}-19$ & 34.61556 \\
\hline KEGG_OOCYTE_MEIOSIS & -0.12728 & -0.02586 & -3.74371 & 0.00021 & 0.002936 & -0.4066 \\
\hline KEGG_CELL_CYCLE & -0.11486 & -0.03202 & -3.45642 & 0.00061 & 0.00793 & -1.40409 \\
\hline KEGG_PROGESTERONE_MEDIATED_OOCYTE_MATURATION & -0.10682 & -0.01851 & -3.00679 & 0.002818 & 0.033812 & -2.81452 \\
\hline
\end{tabular}

TABLE 5 | Gene Set Variation Analysis between high- and low risk groups in the ICGC cohort.

\begin{tabular}{|c|c|c|c|c|c|c|}
\hline NAME & $\log F C$ & AveExpr & $\mathbf{t}$ & P.Value & adj.P.Val & B \\
\hline KEGG_RETINOL_METABOLISM & 0.330529 & 0.056794 & 8.797863 & $2.89 \mathrm{E}-16$ & $5.19 \mathrm{E}-15$ & 26.25868 \\
\hline KEGG_PPAR_SIGNALING_PATHWAY & 0.242533 & 0.034012 & 7.812051 & $1.80 \mathrm{E}-13$ & 3.05E-12 & 19.93605 \\
\hline KEGG_DRUG_METABOLISM_CYTOCHROME_P450 & 0.248736 & 0.027323 & 7.477833 & $1.44 \mathrm{E}-12$ & $2.31 \mathrm{E}-11$ & 17.89126 \\
\hline KEGG_METABOLISM_OF_XENOBIOTICS_BY_CYTOCHROME_P450 & 0.234159 & 0.029967 & 7.336734 & $3.43 E-12$ & $5.14 \mathrm{E}-11$ & 17.0445 \\
\hline KEGG_ARACHIDONIC_ACID_METABOLISM & 0.166018 & -0.00097 & 5.454549 & $1.23 \mathrm{E}-07$ & $1.72 \mathrm{E}-06$ & 6.826222 \\
\hline KEGG_CHEMOKINE_SIGNALING_PATHWAY & 0.087317 & 0.061533 & 3.573573 & 0.000426 & 0.005541 & -0.95374 \\
\hline KEGG_OOCYTE_MEIOSIS & -0.12687 & -0.04236 & -3.35872 & 0.000912 & 0.010941 & -1.66122 \\
\hline KEGG_PROGESTERONE_MEDIATED_OOCYTE_MATURATION & -0.12095 & -0.0516 & -3.26597 & 0.001252 & 0.013767 & -1.9543 \\
\hline KEGG_PATHWAYS_IN_CANCER & -0.05659 & -0.01159 & -3.0279 & 0.002734 & 0.027337 & -2.67212 \\
\hline
\end{tabular}




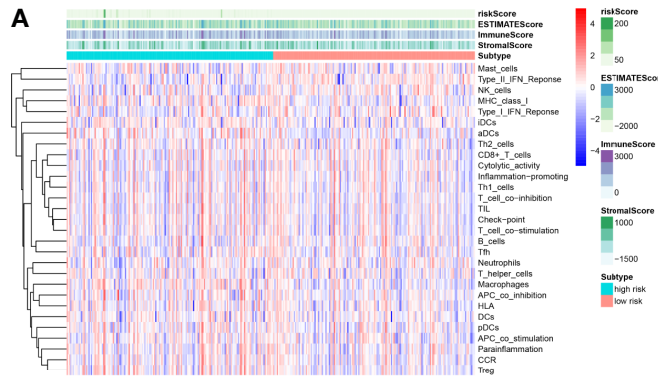

B

Risk 白 low 白 high

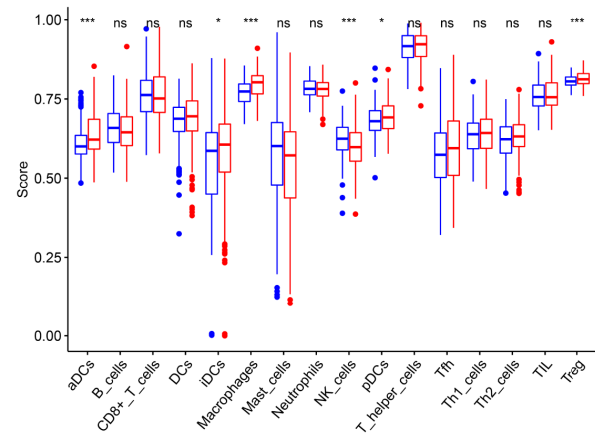

C

Risk 皁 low 帛 high

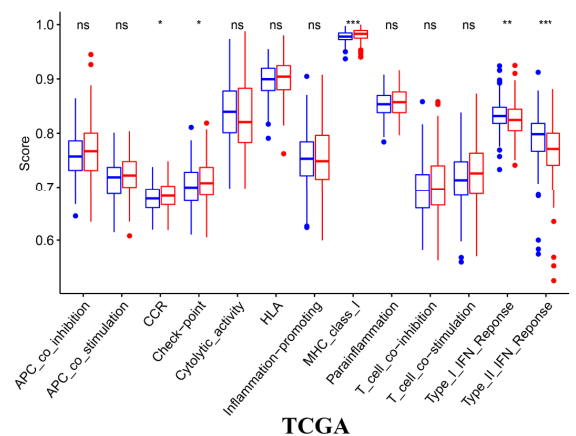

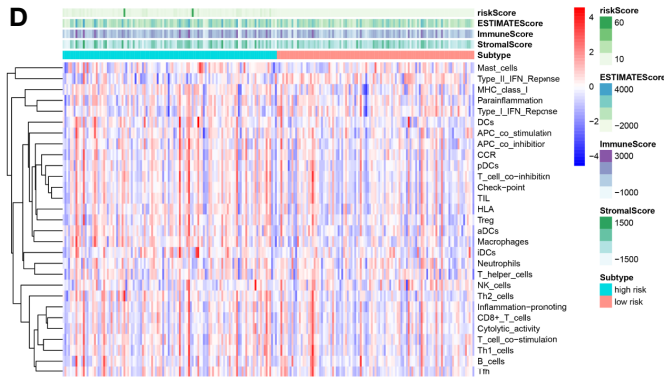

Risk 追 low 追 high

E

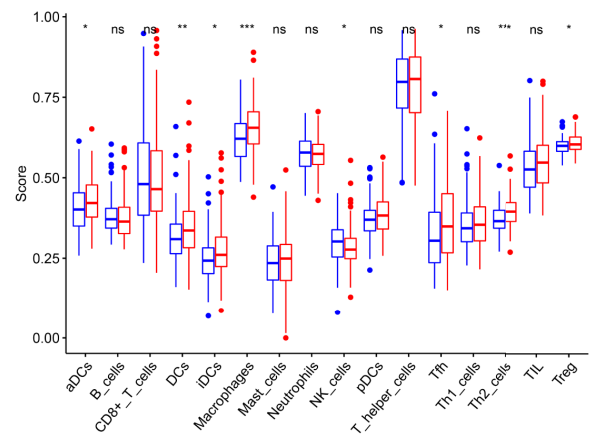

Risk 追 low 白 high

$\mathbf{F}$

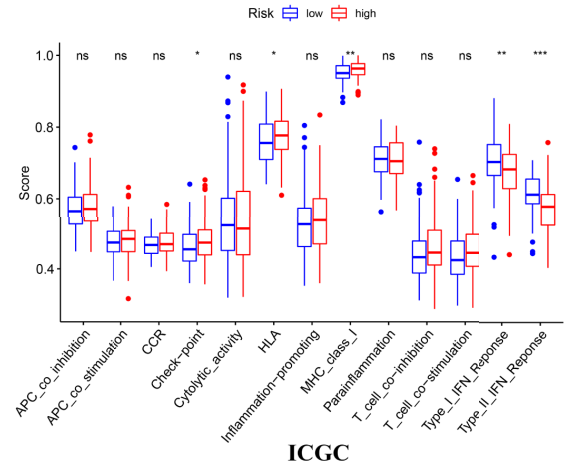

FIGURE 7 The immune landscape between high- and low risk groups in the TCGA and ICGC cohort (A) The heatmap of immune cell infiltration and immune function in the TCGA cohort (B) The differences of immune cell infiltration between high- and low risk groups in the TCGA cohort (C) The differences of immune function between high- and low risk groups in the TCGA cohort (D) The heatmap of immune cell infiltration and immune function in the ICGC cohort (E) The differences of immune cell infiltration between high- and low risk groups in the ICGC cohort (F) The differences of immune function between high- and low risk groups in the ICGC cohort. ${ }^{\star * *} p<0.001$; ${ }^{* *} p<0.01$; ${ }^{*} p<0.05$. ns, no significant.

\section{DISCUSSION}

A majority of HCC patients are at the medium and advanced phases when diagnosed because there are no obvious symptoms in the early stage of HCC (18). Although many therapeutic strategies have been attempted, such as radiofrequency ablation, surgical resection and liver transplantation, the prognostication of HCC cases receiving these treatments is still unsatisfactory (19). Elucidating the molecular mechanism and process of HCC is very important for identifying new therapeutic targets and improving the clinical outcomes of HCC patients.

At present, the traditional methods for predicting prognosis based on a single biomarker or clinical data lack systematic evaluation, resulting in a lack of high sensitivity and specificity. Due to the complex molecular regulation mechanism of HCC, traditional clinical pathological staging cannot fully reflect the heterogeneity of tumors, and the ability to predict prognosis is poor. Therefore, the construction of a gene sequencing prediction model based on big data has great potential for clinical transformation and has become an urgent need to improve clinical efficacy.

The TP53 mutation process is one of the most common mutations in HCC and is considered to be the main driving factor of $\operatorname{HCC}(8,18)$. This gene plays a vital role in maintaining genomic stability, and its loss of function can lead to centrosome amplification, aneuploid cell proliferation and chromosome 


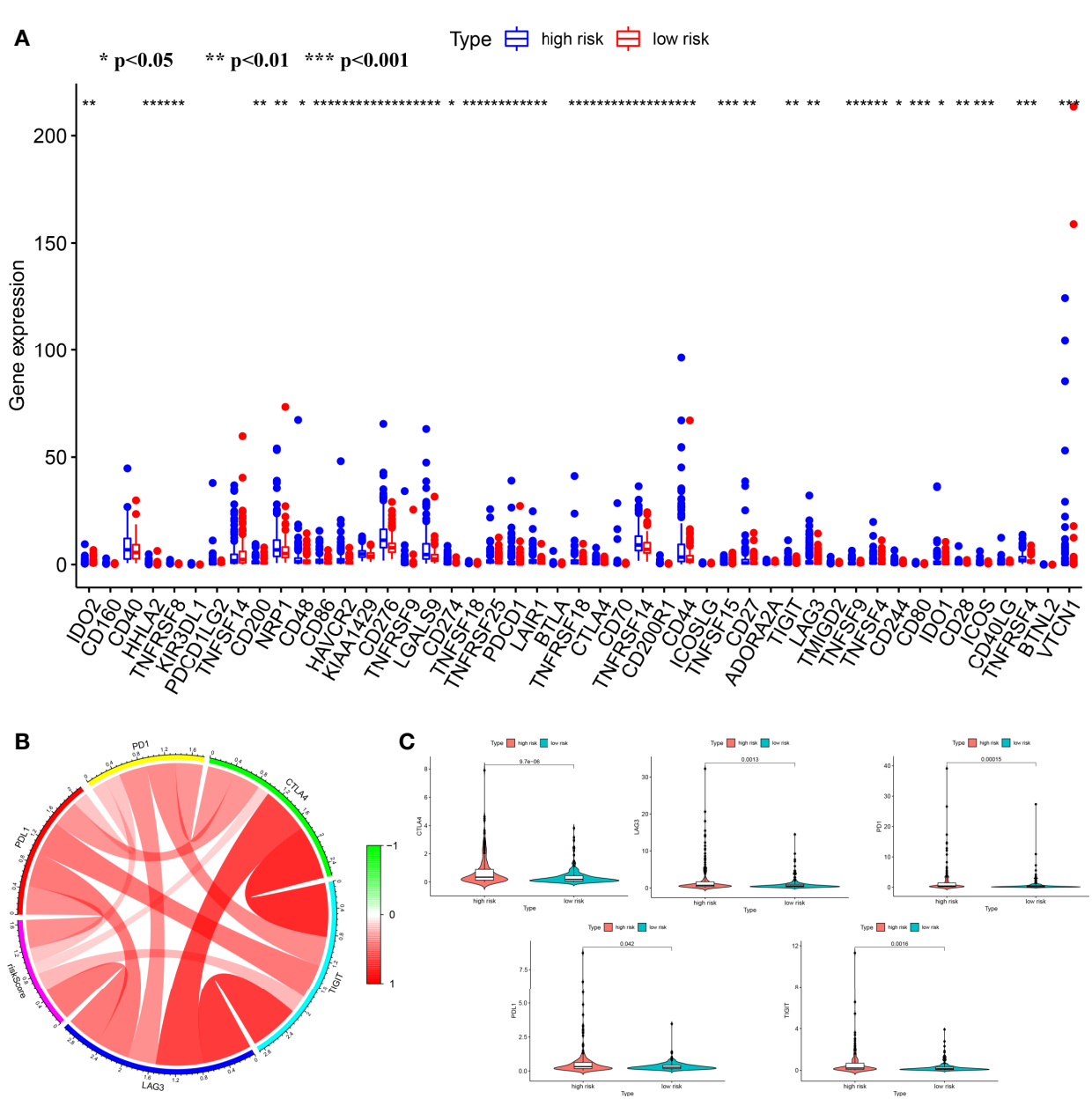

FIGURE 8 | The differences of the expression level of immune checkpoint between high- and low risk groups (A) The boxplot of the expression level of immune checkpoint between high- and low risk groups (B) The correlation analysis between the risk score and the expression levels of PD1, PDL1, TIGIT, CTLA4 and LAG3 $(\mathbf{B}, \mathbf{C})$ The Comparison of the expression level of PD1, PDL1, TIGIT, CTLA4 and LAG3 between high- and low risk groups. ${ }^{\star \star \star} p<0.001$; ${ }^{\star \star} \mathrm{p}<0.01$; ${ }^{\star} \mathrm{p}<0.05$.

instability (20). Compared with TP53 wild-type HCC patients, TP53 mutant HCC patients had shorter overall survival and relapse-free survival (13). We speculated that the poor prognosis of HCC patients with TP53 mutations may be partly due to the change in the RNA expression pattern caused by TP53 mutation. Following this hypothesis, we performed GSEA on HCC samples with and without TP53 mutation. We identified 7 gene sets that were positively correlated with TP53 mutation. After univariate Cox regression analysis, Kaplan-Meier (KM) survival analysis, Lasso penalty Cox regression analysis, and step-by-step multivariate Cox regression analysis, we selected six genes (EIF2S1, SEC61A1, CDC42EP2, SRM, GRM8, TBCD) to construct a prognostic risk score model.

To assess the reliability of the prognostic model, we conducted internal validation, external validation, integrated analysis and subgroup analysis. The AUC values of the ROC curves of 1-, 2-, and 3-year survival of the model were all greater than 0.7 in each independent cohort, which indicated that the signature composed of six genes had good performance in predicting the prognosis of HCC. The proposed signature was superior to a previously TP53-associated prognostic model developed by Long et al. (21) for the assessment of HCC OS.

To explore the regulatory mechanisms of the prognostication model, we performed GSVA on different risk groups. This work indicated that the high-risk cohort exhibited stronger proliferative activity and weaker metabolic activity, which was similar to the results of Gao et al. (22). Based on analysis of the tumor immune microenvironment, the cohort exhibiting high risk showed strong immunosuppression. Because of several important components representing immunosuppression, such as macrophages and Tregs $(23,24)$, the infiltration abundance in the high-risk group was significantly higher than that in the low-risk group. Another important result was that the expression levels of immune checkpoints in the cohort with high risk were significantly higher than those in the cohort with low risk, which was consistent with the positive correlation between the risk score and the 

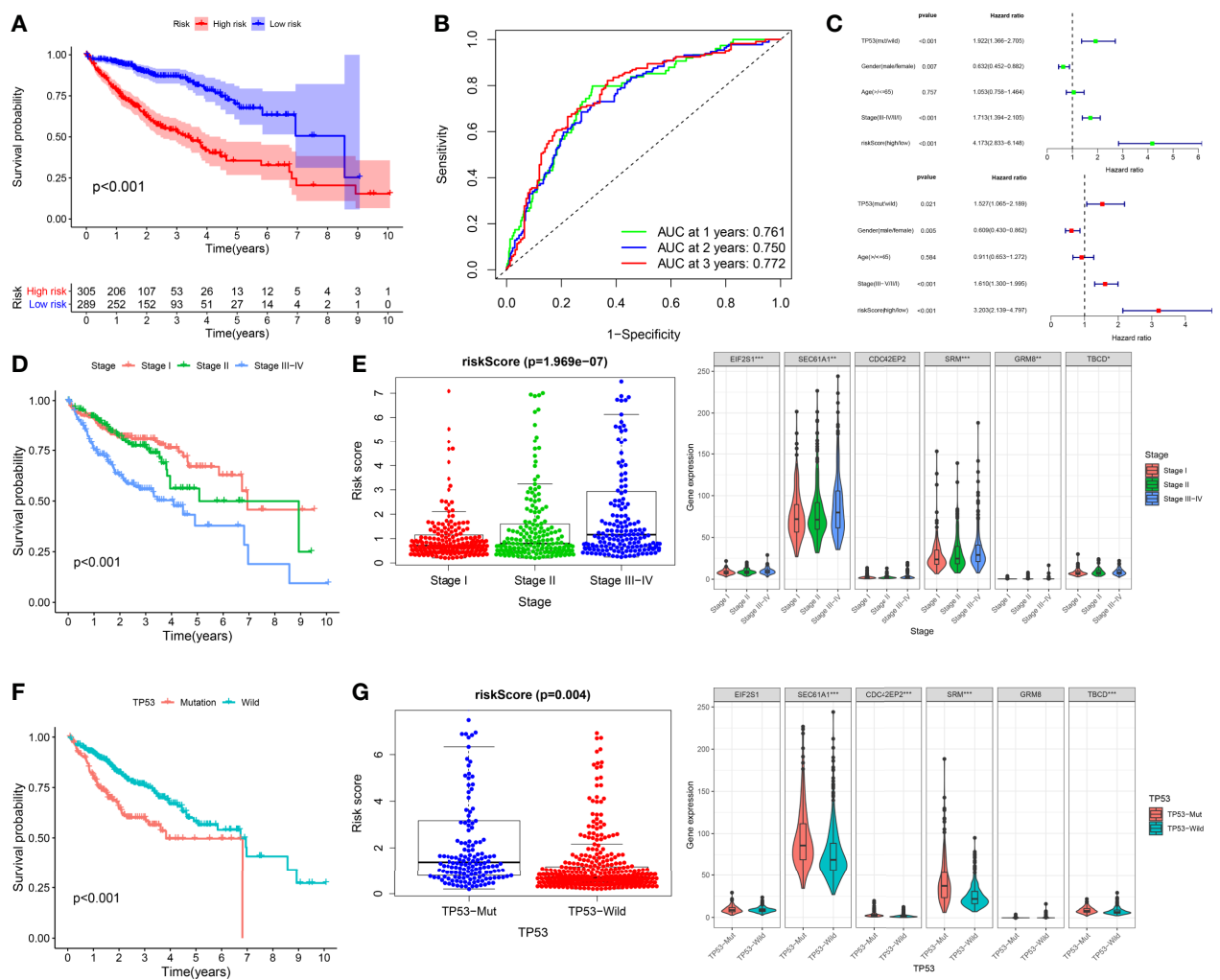

FIGURE 9 | Integrated analysis of the prognostic signature in whole cohort (A-C) The Kaplan-Meier survival analysis, time-dependent ROC analysis, and independence analysis of the signature for predicting the overall survival of patients in the whole cohort( $\mathrm{n}=594)$ (green represents univariate analysis, and red represents multivariate analysis) (D, E) The relationship between the risk score and TNM-stage (F, G) The relationship between the risk score and TP53. ${ }^{\star \star \star} p<$ $0.001 ;{ }^{* *} \mathrm{p}<0.01 ;{ }^{\star} \mathrm{p}<0.05$.

expression level of immune checkpoints. Interestingly, the risk score corresponding to TP53 mutation was also significantly increased. As suggested by Long et al. (25), the immune response of TP53 mutant HCC was significantly weaker. Therefore, we speculated that the poor prognosis of HCC patients in the cohort exhibiting high risk was associated with the tumor microenvironment of immunosuppression. Based on this evidence, we summarized the possible reasons that may lead to weak prognosis in the high-risk group: high proliferative activity, low metabolic activity and immunosuppression.

To date, the six genes in the signature have not been reported in HCC. EIF2S1 participates in premature protein synthesis processes through the formation of a ternary complex with initiators tRNA and GTP (26). SEC61A1 refers to the main polypeptide conduction pathway in the endoplasmic reticulum membrane and the main subunit of the SEC61 complex. Its missense mutation can cause genetic immune-related diseases, such as plasma cell deficiency (27). CDC42EP2 is a member of the CDC42 subfamily that belongs to the Rho family, and the Rho family plays an important role in a variety of cellular processes covering skeletal myogenesis (28). SRM refers to a polyamine biosynthetic enzyme (29). Polyamines modulate the gene expression process through changes in DNA structures and the regulation of signal transduction pathways, and they are consequently associated with proliferation, tumor invasion, and metastasis $(30,31)$. GRM8 pertains to 1 of eight G-protein coupled receptors in the glutamate family and couples to various intracellular second messenger mechanisms to modulate neuronal functions (e.g., neuronal excitability and development) (32). TBCD encodes TBCD (tubulin folding cofactor D), one of five tubulin-specific chaperones that critically impact microtubule assembly in all cells (33). Recently, variants in TBCD have been identified in patients with distinct progressive encephalopathy with an apparently wide clinic-related scope (34).

Our study used TP53 to find 6 new prognostic biomarkers of HCC, and the signature composed of these six genes showed good performance in predicting the prognosis of HCC. However, this work had limits that should be acknowledged. We developed and validated the prognostic risk mode by utilizing general databases, and the outcomes require in-depth confirmation through prospective research. Our major findings were generated from bioinformatics analysis, which demonstrates insufficient verification through in vitro and in vivo experimental processes. In future work, prospective laboratory studies to clarify the specific mechanisms of the six genes in our signature are warranted. 

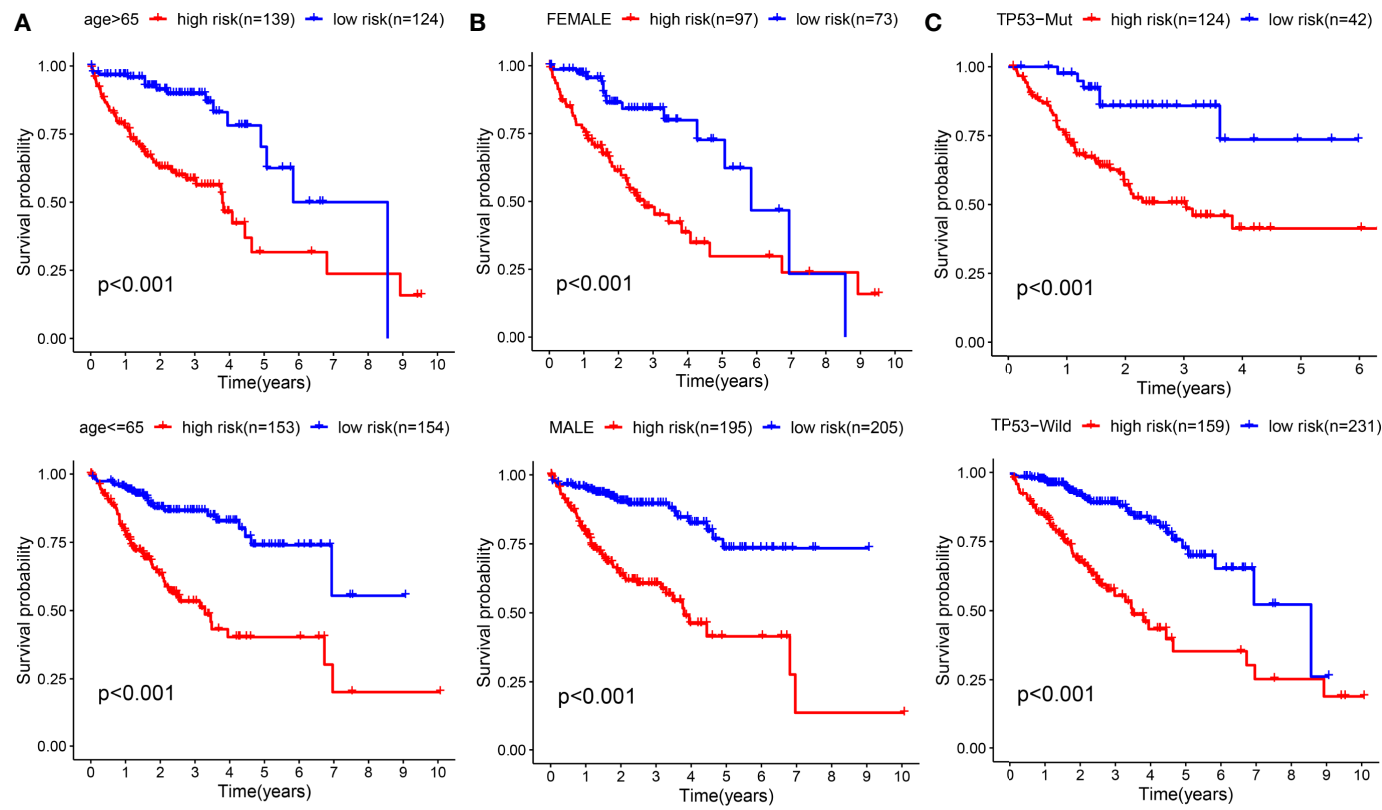

D Stage $\mathrm{I}+$ high risk(n=87) - low risk(n=119)
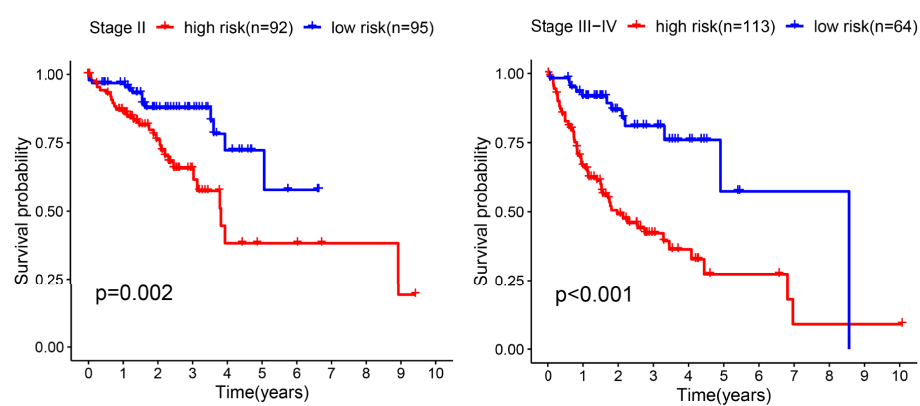

FIGURE 10 | Subgroup Kaplan-Meier survival analysis according to different clinical features in all included HCC patients (A) Age (B) Gender (C) TP53 (D) TNM-stage.

\section{CONCLUSION}

Our study provides a reliable method for the prognostic risk assessment of $\mathrm{HCC}$ and has great potential for clinical transformation.

\section{DATA AVAILABILITY STATEMENT}

Publicly available datasets were analyzed in this study. This data can be found here: The datasets analysed for this study were obtained from The Cancer Genome Atlas (TCGA) (https://

\section{REFERENCES}

1. Bruix J, da Fonseca LG, Reig M. Insights Into the Success and Failure of Systemic Therapy for Hepatocellular Carcinoma. Nat Rev Gastroenterol Hepatol (2019) 16(10):617-30. doi: 10.1038/s41575-019-0179-x

2. Yang JD, Hainaut P, Gores GJ, Amadou A, Plymoth A, Roberts LR. A Global View of Hepatocellular Carcinoma: Trends, Risk, Prevention and Management. Nat Rev Gastroenterol Hepatol (2019) 16(10):589-604. doi: 10.1038/s41575-019-0186-y portal.gdc.cancer.gov/) and International Cancer Genome Consortium (ICGC) (https://icgc.org/).

\section{AUTHOR CONTRIBUTIONS}

$\mathrm{JH}$ designed this study. LW and $\mathrm{YZ}$ contributed to the conception of the study. $\mathrm{JH}$ performed the data analyses and wrote the manuscript. LW helped perform the analysis with constructive discussions. All authors contributed to the article and approved the submitted version. 
6. Donehower LA, Soussi T, Korkut A, Liu Y, Schultz A, Cardenas M, et al. Integrated Analysis of TP53 Gene and Pathway Alterations in the Cancer Genome Atlas. Cell Rep (2019) 28(5):1370-84. doi: 10.1016/j.celrep.2019.07.001

7. Ally A, Balasundaram M, Carlsen R, Chuah E, Clarke A, Dhalla N, et al. Comprehensive and Integrative Genomic Characterization of Hepatocellular Carcinoma. Cell (2017) 169(7):1327-41.e23. doi: 10.1016/j.cell.2017.05.046

8. Rao CV, Asch AS, Yamada HY. Frequently Mutated Genes/Pathways and Genomic Instability as Prevention Targets in Liver Cancer. Carcinogenesis (2017) 38(1):2-11. doi: 10.1093/carcin/bgw118

9. Liu J, Ma Q, Zhang M, Wang X, Zhang D, Li W, et al. Alterations of TP53 Are Associated With a Poor Outcome for Patients With Hepatocellular Carcinoma: Evidence From a Systematic Review and Meta-Analysis. Eur J Cancer (2012) 48(15):2328-38. doi: 10.1016/j.ejca.2012.03.001

10. Park NH, Chung Y-H, Youn KH, Song B-C, Yang SH, Kim JA, et al. Close Correlation of p53 Mutation to Microvascular Invasion in Hepatocellular Carcinoma. J Clin Gastroenterol (2001) 33(5):397-401. doi: 10.1097/00004836-200111000-00011

11. Terris B, Laurent-Puig P, Belghiti J, Degott C, Hénin D, Fléjou JF. Prognostic Influence of Clinicopathologic Features, DNA-Ploidy, CD44H and p53 Expression in a Large Series of Resected Hepatocellular Carcinoma in France. Int J Cancer (1997) 74(6):614-9. doi: 10.1002/(SICI)1097-0215 (19971219)74:6<614::AID-IJC10>3.0.CO;2-5

12. Yuan RH, Jeng YM, Chen HL, Lai PL, Pan HW, Hsieh FJ, et al. Stathmin Overexpression Cooperates With p53 Mutation and Osteopontin Overexpression, and Is Associated With Tumour Progression, Early Recurrence, and Poor Prognosis in Hepatocellular Carcinoma. J Pathol (2006) 209(4):549-58. doi: 10.1002/path.2011

13. Atta MM, El-Masry SA, Abdel-Hameed M, Baiomy HA, Ramadan NE. Value of Serum anti-p53 Antibodies as a Prognostic Factor in Egyptian Patients With Hepatocellular Carcinoma. Clin Biochem (2008) 41(14-15):1131-9. doi: 10.1016/j.clinbiochem.2008.06.006

14. Tu Z, Wu L, Wang P, Hu Q, XZ C, Biology D. N6-Methylandenosine-Related Lncrnas Are Potential Biomarkers for Predicting the Overall Survival of Lower-Grade Glioma Patients. Front Cell Dev Biol (2020) 8:642. doi: 10.3389/fcell.2020.00642

15. Tu Z, Shu L, Li J, Wu L, Huang KJ. A Novel Signature Constructed by RNABinding Protein Coding Genes to Improve Overall Survival Prediction of Glioma Patients. Front Cell Dev Biol (2021) 8:588368. doi: 10.3389/fcell.2020.588368

16. Huo J, Wu L, Zang Y. A Robust Nine-Gene Prognostic Signature Associated With Tumour Doubling Time for Hepatocellular Carcinoma. Life Sci (2020) 260:118396. doi: 10.1016/j.lfs.2020.118396

17. Huo J, Wu L, Zang Y. Development and Validation of a Novel Immune-Gene Pairs Prognostic Model Associated With CTNNB1 Alteration in Hepatocellular Carcinoma. Med Sci Monitor Int Med J Exp Clin Res (2020) 26:e925494-e. doi: $10.12659 / \mathrm{msm} .925494$

18. Rebouissou S, Nault JC. Advances in Molecular Classification and Precision Oncology in Hepatocellular Carcinoma. J Hepatol (2020) 72(2):215-29. doi: 10.1016/j.jhep.2019.08.017

19. Kanwal F, Singal AG. Surveillance for Hepatocellular Carcinoma: Current Best Practice and Future Direction. Gastroenterology (2019) 157(1):54-64. doi: 10.1053/j.gastro.2019.02.049

20. Manning AL, Benes C, Dyson NJ. Whole Chromosome Instability Resulting From the Synergistic Effects of pRB and p53 Inactivation. Oncogene (2014) 33 (19):2487-94. doi: 10.1038/onc.2013.201

21. Long J, Wang A, Bai Y, Lin J, Yang X, Wang D, et al. Development and Validation of a TP53-Associated Immune Prognostic Model for Hepatocellular Carcinoma. EBioMedicine (2019) 42:363-74. doi: 10.1016/j.ebiom.2019.03.022
22. Gao Q, Zhu H, Dong L, Shi W, Chen R, Song Z, et al. Integrated Proteogenomic Characterization of HBV-Related Hepatocellular Carcinoma. Cell (2019) 179 (2):561-77.e22. doi: 10.1016/j.cell.2019.08.052

23. Mitchem JB, Brennan DJ, Knolhoff BL, Belt BA, Zhu Y, Sanford DE, et al. Targeting Tumor-Infiltrating Macrophages Decreases Tumor-Initiating Cells, Relieves Immunosuppression, and Improves Chemotherapeutic Responses. Cancer Res (2013) 73(3):1128-41. doi: 10.1158/0008-5472.CAN-12-2731

24. Stockis J, Liénart S, Colau D, Collignon A, Nishimura SL, Sheppard D, et al. Blocking Immunosuppression by Human Tregs In Vivo With Antibodies Targeting Integrin $\alpha v \beta 8$. Proc Natl Acad Sci USA (2017) 114(47):E10161-8. doi: 10.1073/pnas.1710680114

25. Long J, Wang A, Bai Y, Lin J, Yang X, Wang D, et al. Development and Validation of a TP53-Associated Immune Prognostic Model for Hepatocellular Carcinoma. EBioMedicine (2019) 42:363-74. doi: 10.1016/ j.ebiom.2019.03.022

26. Niso-Santano M, Shen S, Adjemian S, Malik SA, Mariño G, Lachkar S, et al. Direct Interaction Between STAT3 and EIF2AK2 Controls Fatty AcidInduced Autophagy. Autophagy (2013) 9(3):415-7. doi: 10.4161/auto.22910

27. Bolar NA, Golzio C, Živná M, Hayot G, Van Hemelrijk C, Schepers D, et al. Heterozygous Loss-of-Function SEC61A1 Mutations Cause AutosomalDominant Tubulo-Interstitial and Glomerulocystic Kidney Disease With Anemia. Am J Hum Genet (2016) 99(1):174-87. doi: 10.1016/j.ajhg. 2016.05.028

28. Wu M, Yang G, Chen Y, Zhou X, Chen H, Li M, et al. CEP2 Attenuates Myoblast Differentiation But Does Not Affect Proliferation. Int J Biol Sci (2015) 11(1):99. doi: 10.7150/ijbs.8621

29. Casero RA, Marton LJ. Targeting Polyamine Metabolism and Function in Cancer and Other Hyperproliferative Diseases. Nat Rev Drug Discov (2007) 6 (5):373-90. doi: 10.1038/nrd2243

30. Thomas T, TJ T. Polyamine Metabolism and Cancer. J Cell Mol Med (2003) 7 (2):113-26. doi: 10.1111/j.1582-4934.2003.tb00210.x

31. Gerner EW, Meyskens FL. Polyamines and Cancer: Old Molecules, New Understanding. Nat Rev Cancer (2004) 4(10):781-92. doi: 10.1038/nrc1454

32. Stepulak A, Rola R, Polberg K, Ikonomidou CJ. Glutamate and its Receptors in Cancer. J Neural Transm (Vienna) (2014) 121(8):933-44. doi: 10.1007/ s00702-014-1182-6

33. Miyake N, Fukai R, Ohba C, Chihara T, Miura M, Shimizu H, et al. Biallelic TBCD Mutations Cause Early-Onset Neurodegenerative Encephalopathy. Am J Hum Genet (2016) 99(4):950-61. doi: 10.1016/j.ajhg.2016.08.005

34. Grønborg S, Risom L, Ek J, Larsen KB, Scheie D, Petkov Y, et al. A Faroese Founder Variant in TBCD Causes Early Onset, Progressive Encephalopathy With a Homogenous Clinical Course. Eur J Hum Genet (2018) 26(10):151220. doi: 10.1038/s41431-018-0204-5

Conflict of Interest: The authors declare that the research was conducted in the absence of any commercial or financial relationships that could be construed as a potential conflict of interest.

Copyright (c) $2021 \mathrm{Huo}, \mathrm{Wu}$ and Zang. This is an open-access article distributed under the terms of the Creative Commons Attribution License (CC BY). The use, distribution or reproduction in other forums is permitted, provided the original author(s) and the copyright owner(s) are credited and that the original publication in this journal is cited, in accordance with accepted academic practice. No use, distribution or reproduction is permitted which does not comply with these terms. 